\title{
Influence of Cable Temperature Rise on PDC Method Test Results of Water Tree Aged Cables
}

\author{
Jin Ming Guo ${ }^{1}$ and Fei Feng Wang ${ }^{1}$ \\ ${ }^{1}$ Electric Power Research Institute of Guangxi Power Grid Co., Ltd, Nanning 530023, China
}

\begin{abstract}
There is a certain temperature rise in the field running cable because of the load bearing. To study the influence of cable temperature rise on the polarization and depolarization current (PDC) method, $10 \mathrm{kV}$ XLPE short cables were accelerated aging three months by water needle electrode method, and the PDC method was used to test the new and aged cables under constant humidity. The polarization and depolarization current of the cable samples was analyzed, and the conductivity and low frequency dielectric loss spectra were obtained and inspected. The results show that: in the experiment selected test temperature, the change laws of conductivity of the new and aged cables are the same, and the conductivity gradually rose after a slight drop in $40{ }^{\circ} \mathrm{C}$; in the dielectric loss spectrum, the polarization of the new sample is low and the polarization loss changes little with temperature, and the dielectric loss is mainly affected by the conductivity, so the test result is clearly divided into two regions. And water tree aged samples have strong polarization and are obviously affected by temperature, so their low frequency dielectric loss showed the trend of first rising and then decreasing and the maximum appeared at $60^{\circ} \mathrm{C}$.
\end{abstract}

\section{Introduction}

Since the invention of cross-linked polyethylene (XLPE) cable, it has been widely used in China's power grid construction with its excellent insulation performance and mechanical strength. However, with the increase of cable service life, coupled with poor operating environment and heavy load, insulation problems such as water tree aging begin to appear[1,2]. Therefore, rapid and accurate diagnosis of cable water tree aging phenomenon has become one of the important measures to ensure the safe of power grid operation.

As a new testing method based on dielectric response principle, polarization and depolarization current (PDC) method is widely used in transformers, cables and other equipment because of its simple testing process and compact equipment[3-5]. Some scholars have explored the relationship between dielectric polarization time and temperature, and pointed out that with the rise of temperature, the polarization completion time will be shortened[6]. In addition, the time domain response law of insulation under the influence of temperature, the hysteresis phenomenon of PDC and the relationship between time constant and cable insulation state are reported[7-9]. There is a certain temperature rise in the load-bearing cable, and the electrical performance will change accordingly. The influence of temperature on the test results of water tree aging cable insulation by PDC method is a problem worthy of attention. However, there are few relevant literature reports at present.

In this paper, the PDC test of cable at different temperature was carried out based on the accelerated water tree aging cable samples. By studying the changes of conductivity and low frequency dielectric loss spectrum of cable sample, the influence of temperature on PDC test results of cable was investigated.

\section{Sample preparation}

The YJLV223×95 8.7/10kV XLPE cable was selected as the experimental sample, which was cut into a short cable of about $50 \mathrm{~cm}$, and the outer sheath, steel armor and copper shielding layer of the cable sample were removed. In order to facilitate pressurization, one end of the cable was stripped to expose $5 \mathrm{~cm}$ cable core, and the outer semi conductive layer of about $10 \mathrm{~cm}$ was stripped at both ends to prevent surface flashover during pressurization. Then the samples should be sealed to prevent moisture. Finally, both ends of the cable should be wrapped with heat shrinkable tube, and a small opening should be opened in the middle of the heat shrinkable tube to inject $\mathrm{NaCl}$ solution.

The experimental cable samples were divided into two groups. The first group was used as the control sample without any treatment. The second group was the experimental sample. Steel needle with a radius of curvature of $2.5 \pm 0.2 \mathrm{~m}$ were used to drill holes with a depth of about $3 \mathrm{~mm}$ on the outer semiconducting layer evenly and vertically, and saturated $\mathrm{NaCl}$ solution was injected into the heat shrinkable tube. In order to obtain aging cable samples quickly, the high voltage of $7.5 \mathrm{kV}$ and $400 \mathrm{~Hz}$ were applied to the cable insulation. The schematic diagram of experimental aging platform is shown in the Figure 1. 


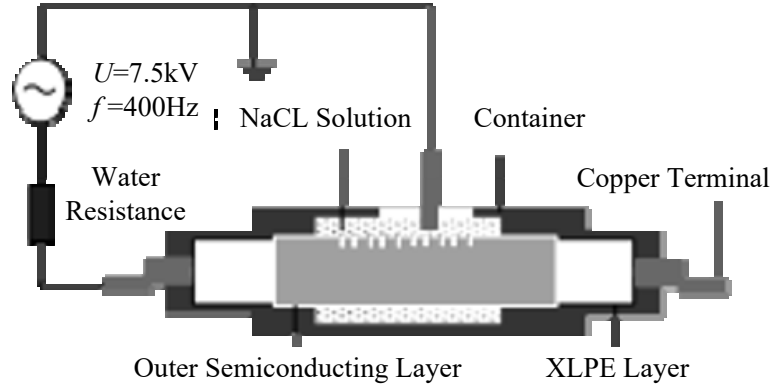

Fig.1 The diagram of cable water tree aging experiment

After 90 days of accelerated aging, all cables in aging group were taken out after PDC test. The insulation near the pinhole was cut into about $100 \mu \mathrm{m}$ thick slices with a slicer, and then dyed in an oven at $90^{\circ} \mathrm{C}$ for half an hour after soaked in methylene blue solution. The surface of the dyed sample was observed by optical microscope. As shown in Figure 2, the typical water tree shape area of the aging sample slice was relatively wide and existed in the form of flakes, which indicated that the aging degree of the cable is deep.
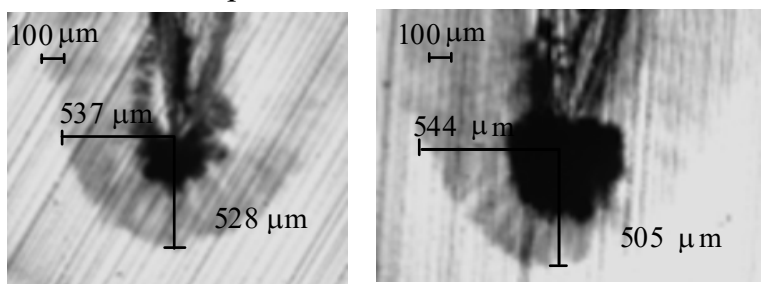

Fig.2 Micromorphology of water tree aged cable samples

\section{Testing procedure}

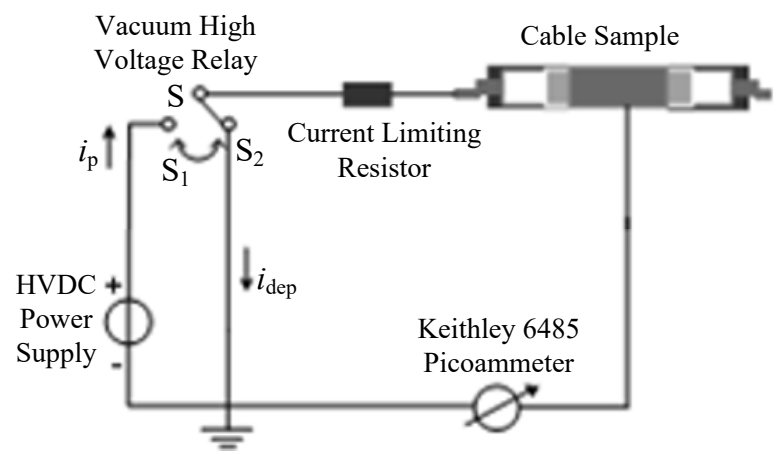

Fig.3 PDC Detection System for XLPE cables

The test wiring is shown in Figure 3. The cable core is connected to the S-end of the switch of the PDC test device. The outer semi conductive layer is covered with copper layer and connected to the picoammeter for grounding. The cable samples were placed in DHG$9070 \mathrm{~A}$ oven during the test. When the temperature inside the oven rose to the test temperature, the cable sample should be kept in it for 2 hours to ensure that the test process was carried out under constant temperature and humidity. Before PDC test, the initial measurement of the tested cable sample was carried out to determine the system bias and the surrounding interference signal without applying polarization voltage. In order to avoid irreversible damage to the cable sample insulation caused by excessive voltage, the applied voltage was $1 \mathrm{kV}$ DC voltage. Considering the limited outage time of the operating cable, and in order to measure the cable sample as accurately as possible, the polarization and depolarization time was set to 90 seconds.

\section{Parameter analysis}

\subsection{Cable Polarization Current}

When the DC voltage is applied to the dielectric, the polarization phenomenon will occur in the dielectric, which is shown as polarization current in macroscopical view. When the applied voltage is removed, the relaxation phenomenon will occur in the dielectric, which is shown as depolarization current in macroscopical view. Theoretically, the polarization current of the cable sample is only a constant DC part more than the depolarization current. The polarization current and depolarization current are the same in the size change trend. Therefore, this paper only shows the polarization current changes of cable samples at different test temperature before and after aging in Figure 4.

After water tree aging, the interface polarization of the cable sample will be strengthened, and the temperature rise will affect the molecular thermal motion of insulation and the polarization of cable. The second half of polarization current is mainly affected by the conductivity of sample, while the first half is affected by both polarization intensity and conductivity.

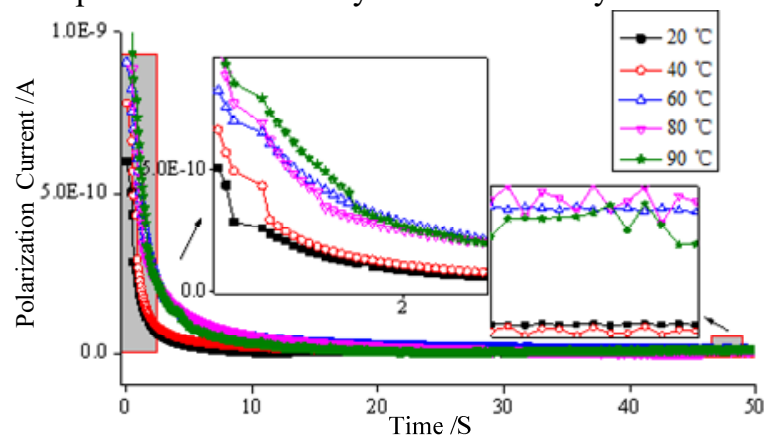

(a) Polarization current of unaged cable sample

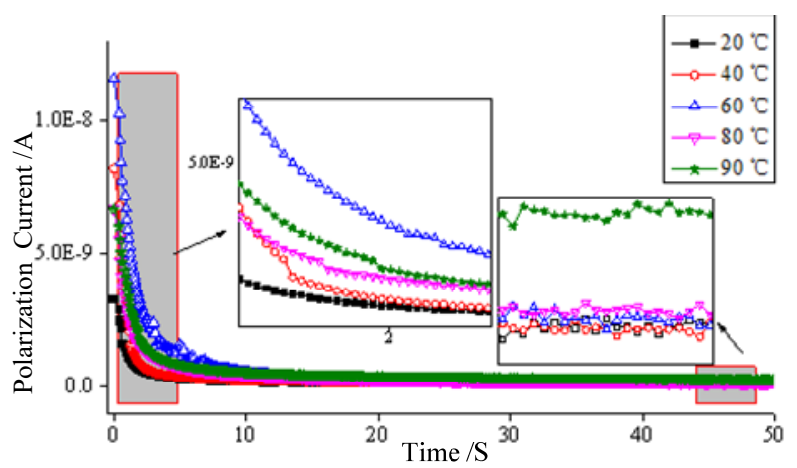

(b) Polarization current of aged cable sample

Fig.4 The PDC results of cable sample at different temperatures

It can be seen from Figure 4 that the polarization current of the unaged sample at different temperatures is obviously divided into two levels in the first half of the 
polarization current, while the polarization current of the aged sample is the largest at $60^{\circ} \mathrm{C}$. With the increase of polarization time, the cable sample gradually began to fully polarize, and the current size was mainly related to the DC conductivity of the sample. At this time, the current of the aging cable sample reached the maximum at $90^{\circ} \mathrm{C}$. It should be noted that the polarization current at $90^{\circ} \mathrm{C}$ was not the maximum at the beginning of the aging process, which indicated that the polarization intensity of the water tree aging cable was inhibited by the increase of temperature. Therefore, in order to comprehensively judge the change of cable insulation performance with temperature, it is necessary to find the quantifiable electrical parameters and study the change trend of it under different test temperatures.

\subsection{Derivation of Electrical Parameter}

It is assumed that the cable is a large ideal flat plate electrode and the XLPE insulation is isotropic. The electrodes are filled with insulating dielectric whose dielectric constant is $\varepsilon_{\mathrm{r}}$. And the transitional inner semiconducting layer is ignored. When the electric field $E$ is applied, the polarization intensity of the cable insulation can be expressed as follows:

$$
\vec{P}(t)=\varepsilon_{0}\left(\varepsilon_{\infty}-1\right) \vec{E}(t)+\varepsilon_{0} \int_{-\infty}^{t} f(t) \vec{E}(t-\tau) d \tau
$$

Where $\varepsilon_{\infty}$ is the high frequency component of the dielectric constant of XLPE insulation, which is equal to the square of the optical refractive index of XLPE material. The first term on the right side of the equation is the instantaneous displacement part, and the second term is the relaxation polarization part. The function $f(t)$ is the response function reflecting the slow polarization behavior of dielectric.

According to Maxwell equation, when the applied voltage is $U(t)$, the total current $i(t)$ flowing through the cable insulation can be expressed as:

$$
i(t)=C_{0}\left[\frac{\sigma_{0}}{\varepsilon_{0}} U(t)+\varepsilon_{\infty} \frac{d U(t)}{d t}+\frac{d}{d t} \int_{-\infty}^{t} f(t) U(t-\tau) d \tau\right]
$$

Where the applied voltage is $U(t)=E(t) \cdot \mathrm{d}, \mathrm{d}$ is the XLPE insulation thickness(the XLPE insulation thickness of $8.7 / 10 \mathrm{kV}$ cable is $4.5 \mathrm{~mm}) . \varepsilon_{0}$ is the vacuum dielectric constant and $\sigma 0$ is the DC conductivity of XLPE insulation[10].

If the cable sample has been fully discharged before charging, the DC power $U_{\mathrm{C}}$ is connected at the time of $t=0$, at this time, the polarization current through the cable insulation layer of the sample can be expressed as:

$$
i_{\text {pol }}=C_{0} U_{0}\left[\frac{\sigma_{0}}{\varepsilon_{0}}+\varepsilon_{\infty} \delta(t)+f(t)\right]
$$

Where the $\delta(t)$ is impulse function. Since it is difficult to accurately measure the impulse change of current amplitude in the process of insulation fast polarization, it is generally not considered. Then the formula (3) can be expressed as:

$$
i_{\text {pol }}=C_{0} U_{0}\left[\frac{\sigma_{0}}{\varepsilon_{0}}+f(t)\right]
$$

When $t=t_{\mathrm{C}}\left(t_{\mathrm{C}}\right.$ is the charging time), the tested cable is short circuited to the ground. At this time, the current flowing through the insulation layer and shielding layer is depolarization current. According to the superposition theorem, it can be equivalent to apply voltage $-U_{\mathrm{C}}$ to XLPE insulation from $t=t_{\mathrm{C}}$, then the depolarization current can be expressed as:

$$
i_{\text {depol }}=-C_{0} U_{0}\left[f(t)-f\left(t+\mathrm{t}_{c}\right)\right]
$$

Since $f(t)$ is monotonic decreasing function, when $t_{\mathrm{C}}$ is large, the second term on the right in formula (5) can be ignored. At this time, it is approximately considered that the depolarization current of the cable is proportional to the dielectric response function of the insulation. There is the following formula:

$$
f(t) \approx \frac{-i_{\text {depol }}(t)}{C_{0} U_{0}}
$$

According to equations (4) and (6), the polarization current can be considered as the sum of depolarization current and conduction current.

When the test time is long enough, the DC conductivity of XLPE insulation can be calculated by solving equations (4) and (6), as shown in formula (7).

$$
\sigma_{0} \approx \frac{\varepsilon_{0}}{C_{0} U_{0}}\left[i_{\text {pol }}(t)+i_{\text {depol }}(t)\right]
$$

Where $C_{0}$ is the vacuum geometric capacitance of power cable insulation.

Assuming that the dielectric response function $f(t)$ of XLPE insulation dielectric obeys the Curie-von Schweidler model, the Fourier transform can be used for simple conversion from time domain to frequency domain[11]. Whether the dielectric response function $f(t)$ is analytic function or two-dimensional array expressed numerically, Fourier transform can be performed[12-16]. Then the current expression in frequency domain can be obtained by Fourier transform of formula (2), as shown in formula (8):

$$
\dot{I}(\omega)=\dot{U}(\omega)\left[\frac{\sigma_{0}}{\varepsilon_{0}} C_{0}+j \omega C_{0}\left(\varepsilon_{\infty}+\dot{F}(\omega)\right)\right]
$$

Since $F(\omega)$ is the Fourier transform of the dielectric response function $f(t)$, the complex polarizability can be expressed as:

$$
\chi(\omega)=\dot{F}(\omega)=\chi^{\prime}(\omega)-j \chi^{\prime \prime}(\omega)
$$

Then the frequency domain expression of the total current can be obtained as follows:

$$
\begin{aligned}
\dot{I}(\omega) & =j \omega C_{0} \dot{U}(\omega)\left[\varepsilon_{0}+\chi^{\prime}(\omega)-j\left(\frac{\sigma_{0}}{\varepsilon_{0} \omega}+\chi^{\prime \prime}(\omega)\right)\right] \\
& =j \omega C_{0} \dot{\varepsilon} \dot{U}(\omega)
\end{aligned}
$$

Therefore, the relationship between the total dielectric loss factor and frequency of cable insulation can be obtained as follows:

$$
\tan \delta(\omega)=\frac{\varepsilon^{\prime \prime}(\omega)}{\varepsilon^{\prime}(\omega)}=\frac{\frac{\sigma_{0}}{\varepsilon_{0} \omega}}{\varepsilon_{\infty}+\chi^{\prime}(\omega)}+\frac{\chi^{\prime \prime}(\omega)}{\varepsilon_{\infty}+\chi^{\prime}(\omega)}
$$

The first term on the right of the equation is conductivity loss of insulation, while the second term is polarization loss, and the sum of the two is total dielectric loss. The DC conductivity $\sigma_{0}$ can be solved by 
formula (7). $\chi^{\prime}(\omega)$ and $\chi^{\prime \prime}(\omega)$ are the real and imaginary parts of the polarizability $\chi(\omega)$ respectively, which can be solved simultaneously by using the depolarization current data through formulas (6) and (9).

Theoretically, the polarization current of cable has only one more constant part of conductance current than the depolarization current, which has no effect on the frequency domain part of the current fast Fourier transform result. Therefore, both the polarization current and depolarization current can be used to calculate the low frequency dielectric loss. However, because of more interference in the polarization process of field power cable, the depolarization current is usually used to calculate the dielectric loss of power cable.

\subsection{Conductivity change}

The conductivity of cable samples measured at different temperatures shown in Figure 5 is calculated by formula (7). It can be seen from the figure that when the temperature is higher than $40^{\circ} \mathrm{C}$, the conductivity of the cable sample increases with the increase of temperature. It should be noted that in the temperature range of $80 \sim$ $90^{\circ} \mathrm{C}$, the conductivity of water tree aging cable sample has a significant jump.

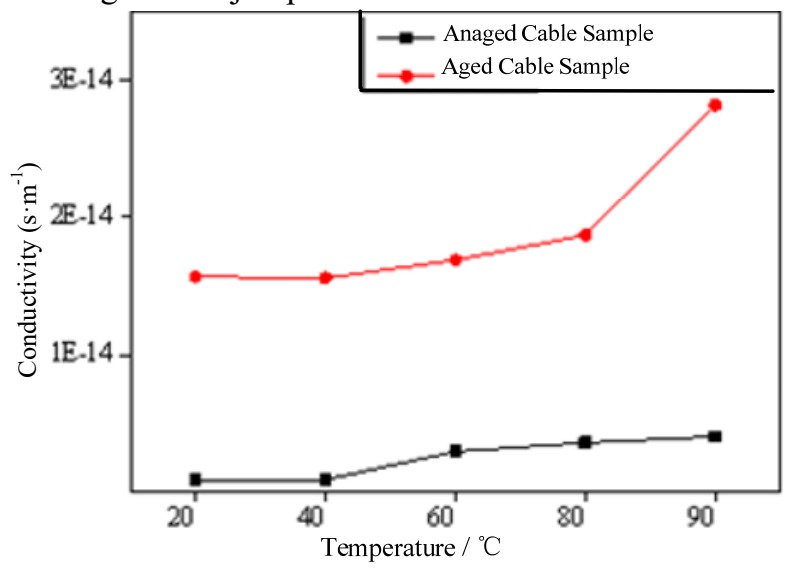

Fig.5 The conductivity change of cable sample with temperature

In order to explain the change of conductivity with temperature, the dynamic thermal mechanical analysis (DMA) test was carried out on the cable slice. DMA is an important method to study the chain motion of polymers and the relationship between structure and property. Figure 6 shows the DMA curve of XLPE sample slice in cable insulation. The storage modulus $\mathrm{E}^{\prime}$ represents the ability of the material to store elastic deformation energy, and its change with temperature will affect the electrical properties of the polymer. The storage modulus decreases with the increase of temperature, which indicates that the molecular thermal motion of insulation will be strengthened with the increase of temperature. When the cable sample is at $60^{\circ} \mathrm{C}$, the XLPE molecular chain will produce $\alpha$ transition, which is related to the relaxation process of crystalline phase moving unit in insulating material, such as the rotation or translation of the lamellar folded chain in the crystalline phase, the sliding between the crystals, and so on. At this time, the conductivity of the cable sample will increase relatively. When the temperature is at $80^{\circ} \mathrm{C}$, the storage modulus of the material will drop to $106 \sim 107 \mathrm{~Pa}$, which indicates that the crystalline phase in XLPE is transforming to amorphous phase $[15,16]$. Therefore, the molecular chain movement will be more intense and further improve the conductivity of the cable sample.

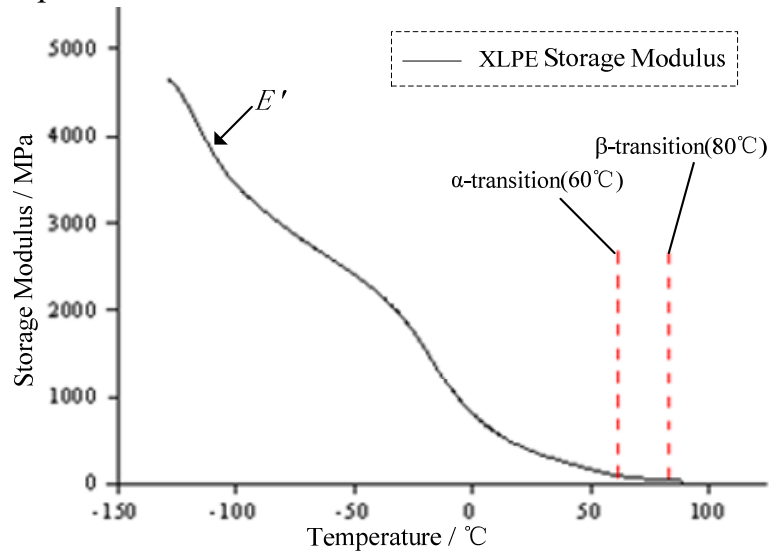

Fig.6 The DMA test curve of cable sample

\subsection{Low frequency dielectric loss spectrum}

Dielectric loss $\tan \delta$ is a comprehensive index to evaluate the dielectric loss characteristics, which only depends on the loss characteristics of the material itself and has nothing to do with other factors. The water tree aging will strengthen the interface polarization of the cable, and the corresponding time is about 0.1 second $\sim$ several hours. Therefore, the selection of low-frequency dielectric loss spectrum can better reflect the interface polarization caused by cable aging [17].

Using formula (11), the low frequency dielectric loss spectrum of cable samples at different temperatures can be obtained, as shown in Figure 7. It can be seen from the figure that the dielectric loss of unaged cable is low, which indicates that its insulation performance is good. At the same time, the dielectric loss of unaged cable can be divided into two levels: $60^{\circ} \mathrm{C}, 80^{\circ} \mathrm{C}$ and $90^{\circ} \mathrm{C}$, which are significantly higher than those of $20^{\circ} \mathrm{C}$ and $40^{\circ} \mathrm{C}$. Due to water erosion, the interface between water tree and insulation presents obvious dielectric stratified. As a result, the polarization and conductivity of the cable sample interface are improved[16]. The temperature will affect the polarization of insulating dielectric, but the molecular thermal motion at over temperature will weaken the polarization. Therefore, under different test temperatures, the dielectric loss of aging cable samples first increases and then decreases, and reaches the maximum at $60^{\circ} \mathrm{C}$. 


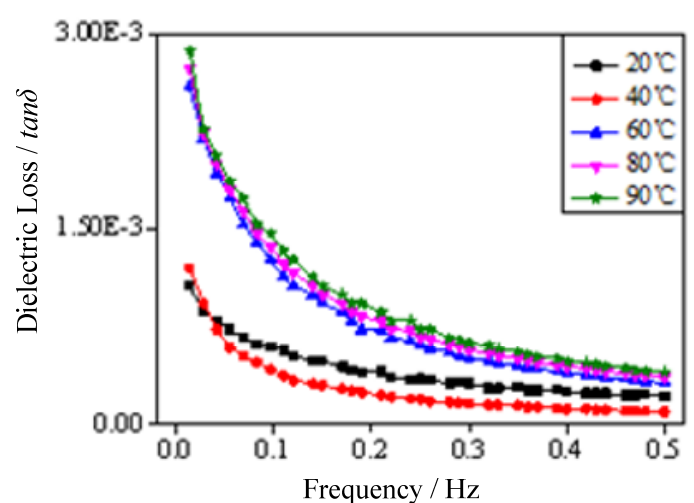

(a) Low frequency dielectric loss spectrum of unaged cable

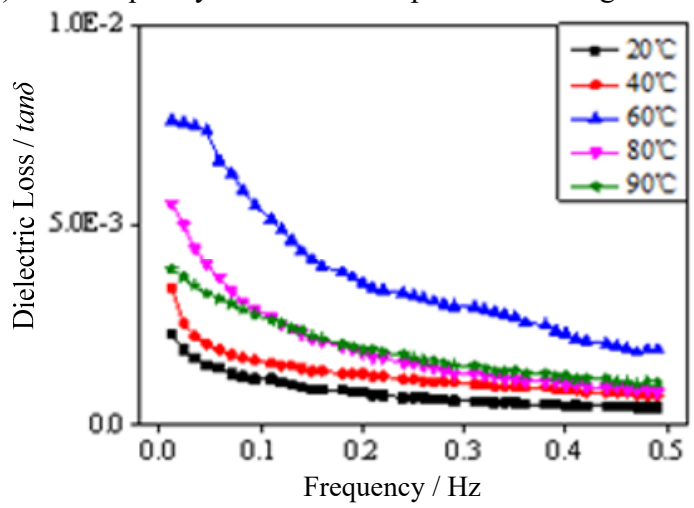

(b) Low frequency dielectric loss spectrum of cables aged for three months

Fig.7 The low frequency dielectric loss of cable samples

\section{Conclusion}

In this paper, PDC method was used to study the variation trend of cable conductivity and low frequency dielectric loss spectrum with temperature, and the results were obtained as follows:

a) With the increase of test temperature, the conductivity of cable samples increased gradually after $40^{\circ} \mathrm{C}$.

b) With the increase of temperature, the dielectric loss of unaged sample was divided into two regions with $60^{\circ} \mathrm{C}$ as the boundary, while the dielectric loss of aging cable samples first increased and then decreased, and reached the maximum at $60^{\circ} \mathrm{C}$.

c) When using PDC method to carry out the test of cable in service, because the temperature has a great influence on the test result, it is suggested that the test should be completed as soon as possible after the cable is shut down.

\section{References}

1. LI Wei, et al. Research Progress in Ageing and Evaluation of XLPE Cable[J]. Insulating Materials, 2016, 49(11): 36-44.

2. Ross R. Inception and propagation mechanisms of water treeing $[\mathrm{J}]$. IEEE Transactions on Dielectrics and Electrical Insulation, 1998, 5(5): 660-680.

3. Yang Wei, et al. Diagnosis of $10 \mathrm{kV}$ XLPE cables insulation aging condition based on the depolarization current method[J]. Electrical Measurement \& Instrumentation, 2015, 52(18): 5-9.

4. Sulaiman S, et al. Simulation modeling of polarization and depolarization current analysis for underground cable insulation[C]. Power Engineering and Optimization Conference (PEOCO), 2013 IEEE 7th international. IEEE, 2013: 358-361.

5. Saha T K, Purkait P. Understanding the impacts of moisture and thermal ageing on transformer's insulation by dielectric response and molecular weight measurements[J]. IEEE Transactions on Dielectrics and Electrical Insulation, 2008, 15(2): 568-582.

6. Koch Maik, Tenbohlen Stefan. Diagnostics of oil paper insulation using relaxation currents[C]. Proceedings of the 14th International Symposium on High Voltage Engineering, Beijing, China, 2005: 112-121.

7. Saha T K, Purkait P. Impact of the condition of oil on the polarization based diagnostics for assessing the condition of transformers insulation[C]. IEEE Power Engineering Society General Meeting. Toronto, Canada: IEEE, 2005: 1882-1886.

8. Fofana I, et al. Effect of thermal transient on the polarization and depolarization current measurements of oil-paper insulation[J]. IEEE Transactions on Dielectrics and Electrical Insulation, 2011, 18(2): 513-520.

9. Talib M A, et al. Diagnosis of transformer insulation condition using recovery voltage measurements[C]. National Power and Energy Conference 2003 Proceedings. Bangi, Malaysia: 2003: 329-332.

10. Yang Yan, et al. Characteristic Parameters for Assessing Aging Condition of Oil-paper Insulation by Polarization/Depolarization Current[J]. High Voltage Engineering, 2013, 39(2): 336-341.

11. Farahani M, Borsi H, Gockenbach E. Dielectric response studies on insulating system of high voltage rotating machines[J]. IEEE Transactions on Dielectrics and Electrical Insulation, 2006, 13(2): 383-393.

12. Helgeson A, Gafvert U. Dielectric response measurements in time and frequency domain on high voltage insulation with different response[C]. IEEE International Symposium on Electrical Insulating Materials, 1998: 393-398.

13. Mopsik F I. Relaxation. The quantitative application of time-domain techniques to dielectrics[J]. IEEE Transactions on Dielectrics and Electrical Insulation, 2002, 9(5): 829-837.

14. Farag N, et al. Numerical transformations of widerange time- and frequency-domain relaxational spectra[J]. IEE Proceedings-Science, Measurement and Technology, 2003, 150(2): 65-74.

15. Bower D I. An Introduction to Polymer Physics[M]. New York: Cambridge University Press, 2002: 216.

16. Zhu Cheng-shen. Analysis of Polymer Structure[M]. Beijing: Science Press, 2004: 157-160, 181, 214-224. 
17. Liu Ying, et al. Ageing Condition Assessment of XLPE Cable Insulation Based on tan $\delta$ at $0.1 \mathrm{~Hz}[\mathrm{~J}]$. Insulating Materials, 2016, 49(05): 41-45. 